\title{
El video como herramienta TIC en inglés aplicada en el medio superior de la Universidad Autónoma de Campeche
}

\section{Video as an ICT tool in English applied in the high school of the Universidad Autónoma de Campeche}

DOI: $10.46932 / \mathrm{sfjdv} 3 \mathrm{n} 1-109$

Received in: Jan 30st, 2021

Accepted in: Feb 1th, 2022

\author{
Mayté Cadena González \\ Maestra en Educación Superior \\ Universidad Autónoma de Campeche \\ Avenida Agustín Melgar s/n Col. Buenavista CP. 24039, \\ San Francisco de Campeche, Campeche. México. \\ E-mail:macadena@uacam.mx \\ María Alejandra Sarmiento Bojórquez \\ Doctora en Educación \\ Avenida Agustín Melgar s/n Col. Buenavista CP. 24039, \\ San Francisco de Campeche, Campeche. México. \\ E-mail:masarmie@uacam.mx

\section{Juan Fernando Casanova Rosado} \\ Doctor en Educación \\ Avenida Agustín Melgar s/n Col. Buenavista CP. 24039, \\ San Francisco de Campeche, Campeche. México. \\ E-mail: jfcasano@uacam.mx
}

\begin{abstract}
RESUMEN
El avance tecnológico hoy en día está inmerso en todo, incluyendo la educación, la cual debe seguir incorporando todas las TIC en sus estrategias de aprendizaje. Un recurso muy utilizado por los jóvenes para comunicarse en todo es el video, siendo este un excelente medio para aprender un nuevo idioma; en el nivel medio superior la unidad de aprendizaje de inglés en todos sus semestres evalúa la comprensión oral y escrita, es una herramienta perfecta para evaluar.

El objetivo del estudio es determinar si el alumno considera el elaborar videos como herramienta TIC como medio de enseñanza del idioma inglés en el nivel medio superior de la Universidad Autónoma de Campeche.

Un cuestionario autoadministrable fue aplicado a 86 sujetos. De estos hay un promedio de edad $\mathrm{x}=17.06$ y una DS $=.725$, Sobre si consideran la elaboración del video como herramienta útil, el $48.8 \%$ (42 sujetos) manifestaron estar de acuerdo, el 31.4\% (27 sujetos) dicen estar totalmente de acuerdo, solo el $4.7 \%$ (4 sujetos) estuvieron en desacuerdo. También consideran que elaborar videos ayuda para el aprendizaje del idioma inglés, 45 sujetos (52.3\%) manifestaron estar de acuerdo; 26 sujetos (30.2\%) dijeron estar totalmente de acuerdo, solo 4 sujetos $(4.7 \%)$ manifestaron estar en desacuerdo.

En conclusión, los estudiantes están de acuerdo que, al realizar videos en inglés utilizamos las TIC y estas ayudan a aprender el idioma, manifiestan que es una herramienta útil y una tecnología de información
\end{abstract}


interactiva, permitiendo procesos de aprendizaje autónomos que consolidan los principios de "aprender a aprender".

Palabras clave: TIC, inglés, video, recurso didáctico.

\begin{abstract}
Technological progress today is immersed in everything, including education, which must continue to incorporate all ICTs in their learning strategies. A resource widely used by young people to communicate in everything is video, this being an excellent means of learning a new language. At the upper middle level, the English learning unit in all its semesters evaluates oral and written comprehension, it is a perfect tool to evaluate.

The objective of the study is to determine if the student considers making videos as an ICT tool as a means of teaching the English language at the upper secondary level of the Autonomous University of Campeche. A self-administered questionnaire was applied to 86 subjects. Of these, there is an average age $\mathrm{x}=17.06$ and a $\mathrm{SD}=.725$. Regarding whether they consider the making of the video as a useful tool, $48.8 \%$ (42 subjects) said they agree, $31.4 \%$ (27 subjects) say they totally agree. agreed, only $4.7 \%$ (4 subjects) disagreed. They also consider that making videos helps to learn the English language, 45 subjects (52.3\%) agreed; 26 subjects $(30.2 \%)$ said they totally agreed, only 4 subjects $(4.7 \%)$ said they disagreed.

In conclusion, the students agree that, when making videos in English, we use ICTs and these help to learn the language, they state that it is a useful tool and an interactive information technology, allowing autonomous learning processes that consolidate the principles of "learn to learn".
\end{abstract}

Keywords: ICT, English, video, teaching resource.

\title{
1 INTRODUCCIÓN
}

Hoy la mayoría de los estudiantes tienen contacto, conocen y usan los medios informáticos para el desarrollo de tareas escolares, esta tecnología se puede aprovechar para el aprendizaje académico. Hablar de recursos de aprendizaje en la actualidad necesariamente nos lleva al uso de las nuevas tecnologías. Ya quedo en el pasado la entrega de tareas solo en papel, nuestros alumnos tienen un abanico de posibilidades para poder aprender, no solo repitiendo y memorizando, ahora pueden ver, tocar, escuchar, etc. El profesor tiene que hacer uso de los recursos didácticos que nos rodean y que los alumnos conocen. La utilización de los recursos didácticos debe consistir en un proceso sistematizado que facilite la interpretación de los contenidos que se han de enseñar. Los recursos pueden convertirse en verdaderos instrumentos del pensamiento, de innovación, de motivación del aprendizaje, ... facilitando la enseñanza. Es por eso por lo que el video es uno de los recursos de aprendizaje que se utilizan para el estudio del idioma inglés.

Los estudiantes usan a diario los medios informáticos para el desarrollo de tareas escolares, hoy el uso de la tecnología por los jóvenes es una realidad que en ocasiones hasta rebasa al propio docente. El video no es un recurso nuevo, se ha utilizado desde décadas anteriores como un recurso de enseñanza, sin embargo, resultaba con un alto costo el poder realizarlo, pero ahora con la nueva tecnología al alcance de 
todos, desde una computadora de escritorio hasta un simple teléfono celular, cualquiera puede realizar un video. Estas tecnologías aunadas con las habilidades de los alumnos se pueden aprovechar para el aprendizaje académico. La unidad de aprendizaje de inglés evalúa tanto la gramática del idioma como la pronunciación, es decir comprensión oral y escrita, por lo cual el video es una herramienta perfecta para evaluar el desempeño del estudiante.

En esta investigación tienen como objetivo establecer si el alumno considera el video como un recurso de aprendizaje para el estudio de inglés como lengua extranjera. Se considera de suma importancia el poder utilizar la tecnología para el proceso de enseñanza-aprendizaje.

\subsection{EL APRENDIZAJE UTILIZANDO EL VÍDEO.}

Estamos en pleno siglo XXI y es una necesidad el adiestramiento profesional de los docentes en TIC, en la producción de contenidos y materiales educativos, así como en nuevos modelos pedagógico. Para ello, los docentes deben estar sensibilizados hacia la alfabetización digital con el fin de que se apropien de esta nueva cultura y puedan no solo intervenir sus currículos, sino ser parte de redes de conocimiento para asegurar el trabajo grupal y colaborativo, como un servicio público y gratuito que colabora con las políticas educativas nacionales hacia una plataforma tecnológica de acceso libre.

Dentro de los Estándares de Competencia en TIC que maneja la Organización de las Naciones Unidas para la Educación, la Ciencia y la Cultura (UNESCO, 2008, p.2) se menciona que los docentes se deben preparar para empoderar a los estudiantes con las ventajas que les aportan las TIC. Se debe contar con docentes preparados en uso de TIC en cada escuela y cada aula, ya sean presenciales o virtuales. Los docentes deben poseer las competencias y los recursos necesarios en materia de TIC y así poder enseñar de manera eficaz las asignaturas exigidas, integrando al mismo tiempo en su enseñanza conceptos y habilidades de éstas.

Se debe dejar atrás en modelo tradicionalista donde se utilizaba el método conductista y crear nuevos escenarios para el aprendizaje. Desde una perspectiva constructivista a los estudiantes se les propone una tarea inspirada en situaciones reales, la cual deben desarrollar a lo largo del curso en la medida que van alcanzando los objetivos preestablecidos y es ahí donde deben utilizar una gran gamma de recursos de aprendizaje. En este rango se encuentran tanto los videos como los objetos de aprendizaje que ayudan a una mejor adquisición de conocimiento (Del Moral \& Cerne, 2004, p.7).

Las TIC se han introducido en la vida cotidiana: celulares, tablets, skype, ipods, podcast, DVD, televisión digital, twitter, whatsapp, mensajes de texto, ipads, apps.... Difícilmente se encuentre un miembro de la sociedad que no emplee en menor o mayor grado estos recursos. "Al trabajar con tecnologías de la información no es solo utilizar modernos aparatos tecnológicos con el afán de 
actualizarse en el ámbito social, al contrario de esto, es utilizar los recursos tecnológicos para una meta especial la cual es obtener una educación de calidad" (Almeida Aguilar, et al, 2017, p.3). Entonces si el aula refleja la sociedad en que vivimos, es de esperarse que las TIC tengan un papel protagónico o al menos que existan en las clases de inglés en la escuela.

\subsection{EL VIDEO COMO RECURSO DIDÁCTICO.}

Los recursos didácticos juegan un papel muy importante dentro del aula, en el momento del proceso enseñanza-aprendizaje; entendemos que un recurso didáctico es: cualquier material que maestros o alumnos elaboren, seleccionen y utilicen para apoyar los procesos de enseñanza y de aprendizaje (Grisolía, 2015). Es interesante observar cómo al sugerir a los estudiantes la realización de un video, representa un reto doble, tanto por el resultado visual como por el reto de hacerlo en un segundo idioma.

El uso del vídeo ofrece a los alumnos de un segundo idioma, la oportunidad de utilizar el lenguaje en contextos pertinentes, ser creativo, hacer uso del valor de trabajar en equipo, mejorar sus habilidades en relación con la expresión oral y fluidez, y con esto obtener una mayor comprensión para lograr un aprendizaje significativo (Martínez, 2003).

Considerando que el texto y las imágenes, son herramientas fundamentales de la enseñanza y aprovechando que la imagen tiene un poder incuestionable de comunicación, y que bien combinados lo multiplicarían. Definimos al video desde su origen etimológico, esta palabra proviene del latín "videre" y significa "yo veo" (DLE, 2017). En una definición real, el video es una secuencia de imágenes, que crea en el observador una sensación de movimiento, lo cual se refleja, al percibir que transmite y provoca en el espectador emociones y sensaciones.

Sin lugar a dudas los adolescentes en el nivel medio superior, pasan por momentos en que los videos para ellos son atractivos e interesantes, (la mayoría utiliza el celular para observar videos), "Los medios audiovisuales son simples canales mediante los cuales se comunica cualquier contenido (son instrumentos tanto para la recepción del mensaje como para su transmisión)" (Bartolomé, 1987) de ahí la importancia del uso del video en el aula, el cual servirá como transmisor y los alumnos como receptores principales de la información que les permitirá dotarse de ésta, para la construcción de opiniones en torno a determinado tema, tomando además en consideración que tanto como la vista como el oído son los dos sentidos por los cuales el individuo adquiere casi todas sus experiencias, por lo que su uso en el aula permitirá que el alumno desarrolle habilidades y destrezas, necesarias en su vida cotidiana.

El uso del video en el aula va a depender en gran medida de la creatividad e interés del docente, considerando los propósitos de aprendizaje y suscitando el interés sobre los temas a abordar, así el docente podrá determinar según las necesidades de sus alumnos que video requerirá y que función desempeñarán, 
ya que el uso de vídeos ofrece variedad, desarrolla la comprensión, es un soporte muy cercano para los alumnos, permite trabajar aspectos lingüísticos en contextos, llevando la vida real al aula (Corpas, 2004).

De igual o mayor importancia son las interacciones ocurridas entre el alumno y las personas que lo rodean, por tal motivo no se puede evadir el análisis de la influencia educativa que provoca la convivencia del docente, el alumno y sus compañeros de grupo.

Para María Isabel (Blanco Sánchez, 2012) "los recursos de aprendizaje cumplen una función mediadora entre la intencionalidad educativa y el proceso de aprendizaje, entre el educador y el educando", por lo cual es necesario mantener esta relación entre estos dos protagonistas del proceso.

\section{METODOLOGÍA}

El trabajo de investigación corresponde a un estudio cuantitativo de tipo descriptivo no experimental, cuyo instrumento de recolección de datos fue un cuestionario autoadministrable. El lugar donde se realizó la investigación es en la Escuela Preparatoria Dr. Nazario Víctor Montejo Godoy de la Universidad Autónoma de Campeche (UAC). En el estudio participaron los alumnos de quinto semestre de los grupos A, B, C, D y E. A estos alumnos se le solicito realizar un video con su celular representando una escena cotidiana que involucre el uso del vocabulario de la Unidad de aprendizaje correspondiente.

\subsection{PREGUNTA DE INVESTIGACIÓN:}

¿Es la elaboración del video una herramienta TIC en la enseñanza del idioma inglés en el nivel medio superior de la UAC?

Objetivo:

Determinar si la elaboración del video es una herramienta TIC en la enseñanza en inglés aplicada en el nivel medio superior de la Universidad Autónoma de Campeche. Método:

Se elaboró un cuestionario (autoadministrable) de 17 preguntas cuya elaboración fue apoyada en un consenso de expertos. Se seleccionó una muestra de alumnos del turno matutino de la Esc. Prep. Dr. Nazario Víctor Montejo Godoy de nivel medio superior para aplicarles el cuestionario. Los sujetos de investigación en su clase de inglés elaboran videos como proyectos finales y parciales, las características principales es que sean cortos, usen la gramática esperada, el vocabulario visto en las lecciones, y es una herramienta donde se puede evaluar las 4 habilidades del aprendizaje de una lengua extranjera. Ellos usan su creatividad y diseño propio. Realizan sus videos en equipo desarrollando competencias colaborativas.

El cuestionario fue aplicado a 86 sujetos (alumnos) de la UAC, que cursan la Educación Media Superior, en el turno matutino; siendo distribuido por un investigador el cual explicó la manera de llenar los 17 items. 
Se recolectaron los cuestionarios y se elaboró una base de datos la cual fue analizada en el programa estadístico S.P.S.S. versión 15.

\section{RESULTADOS Y DISCUSIÓN}

Un total de 86 sujetos fueron encuestados con un promedio de edad $\mathrm{x}=17.06 \mathrm{y}$ una DS $=.725$, de 105 cuales 31 sujetos (36\%) fueron hombre y 55 sujetos (64\%) fueron mujeres.

En cuanto a su nivel de conocimientos de inglés se observó lo siguiente: en el nivel básico 56 sujetos $(65.1 \%)$ en intermedio 22 sujetos $(25.6 \%)$, solo 3 (3.5\%) sujetos estuvieron en el grupo de nivel avanzado y para nivel nulo fueron 5 sujetos $(5.8 \%)$.

De la promoción de la elaboración de videos por el profesor, el 44\% (37 sujetos) opinaron estar de acuerdo, el 42.9\% (36 sujetos) estuvieron totalmente de acuerdo y solo el 7.1\% (6 sujetos) manifestaron estar en desacuerdo y 7 son indiferentes.

Para la pregunta sobre si consideran la elaboración del video como una herramienta útil, el 48.8\% (42 sujetos) manifestaron estar de acuerdo, el 31.4\% (27 sujetos) manifestaron estar totalmente de acuerdo, solo el $4.7 \%$ (4 sujetos) estuvieron en desacuerdo y 13 no se manifestaron.

En cuanto a la pregunta sobre si consideraban que elaborar videos ayuda para el aprendizaje del idioma Ingles, 45 sujetos (52.3\%) manifestaron estar de acuerdo; 26 sujetos (30.2\%) dijeron estar totalmente de acuerdo, solo 4 sujetos (4.7\%) manifestaron estar en desacuerdo y 11 sujetos (12.8\%) son indiferentes. (Figura 1)

Figura 1. La elaboración del video como ayuda en el aprendizaje de inglés.

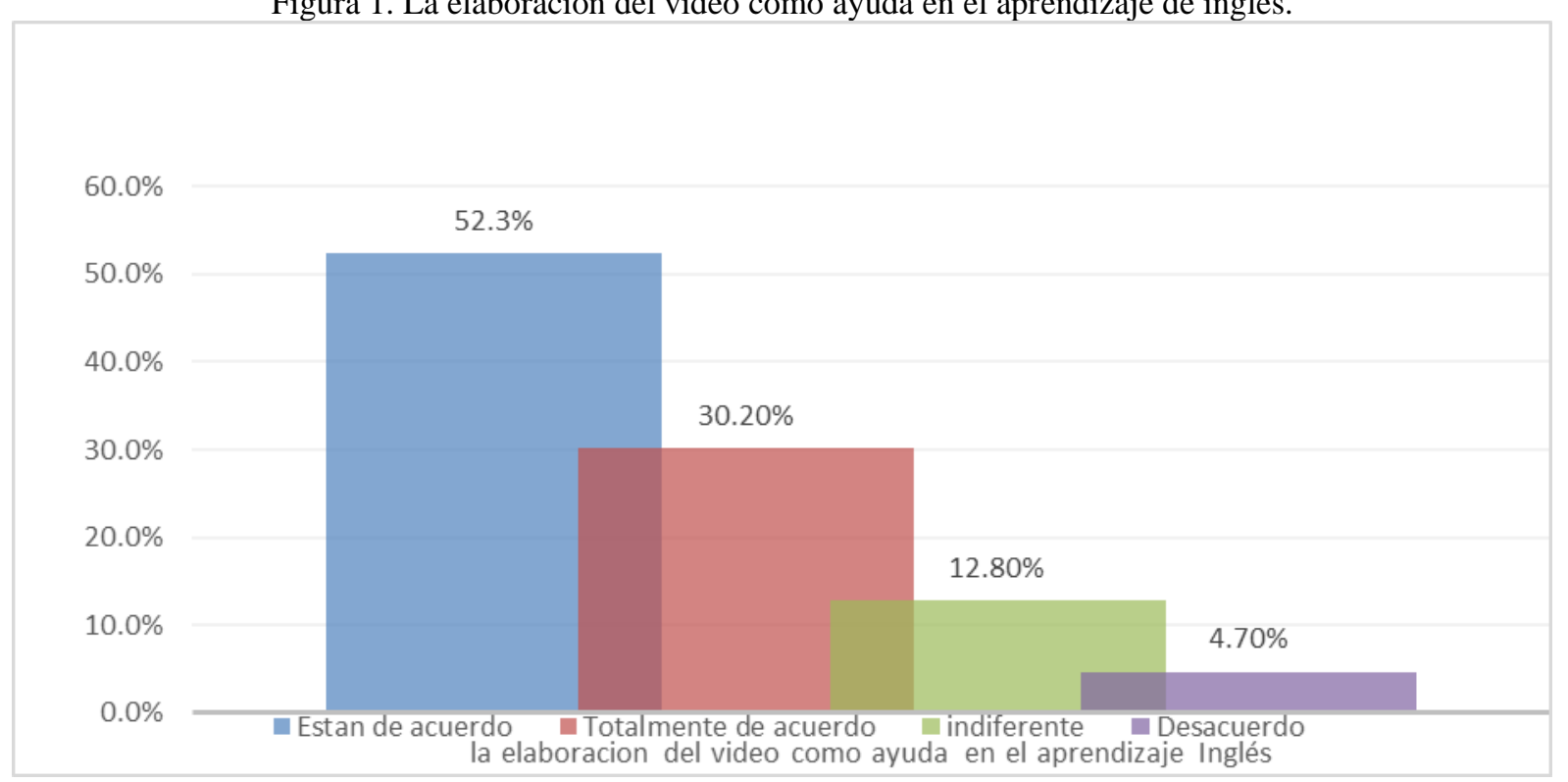


La pregunta sobre que Sí al realizar un video en ingles practico mi pronunciación el 51.2\% (44sujetos) manifestaron estar totalmente de acuerdo, 44.2\% (38 sujetos) dijeron estar de acuerdo, 3.5\% (3 sujetos).

Cuando se les pregunto si al realizar un video practican su vocabulario el 51.2\% (44sujetos) dijeron estar de acuerdo; $38.4 \%$ (33 sujetos) manifestaron estar totalmente de acuerdo; mientras que solo el 1.2\% ( 1 sujeto) manifestó estar en desacuerdo y $9.2 \%$ (8 sujetos) son manifestaron ser indiferentes y 1.2\% (1 sujeto) manifestó estar en desacuerdo.

Al preguntarles sobre si al realizar un video practican su gramática; el 55.8\% (48 sujetos) mencionaron estar de acuerdo seguido por el $26.7 \%$ (23 sujetos) dijeron estar totalmente de acuerdo; aun así, el 2.3\% (2 sujetos) manifestaron estar en desacuerdo.

Para la pregunta al realizar un video me da seguridad para expresarme en inglés, 36 sujetos el (41.9\%) mencionó estar de acuerdo, 26 sujetos (30.2\%) dijeron ser indiferentes, 21 sujetos (24.4\%) manifestaron estar totalmente de acuerdo, y solo 3 sujetos (3.5\%) manifestaron estar en desacuerdo. (Figura 2)

Figura 2. Realizar un video ¿da seguridad para expresarme en inglés?

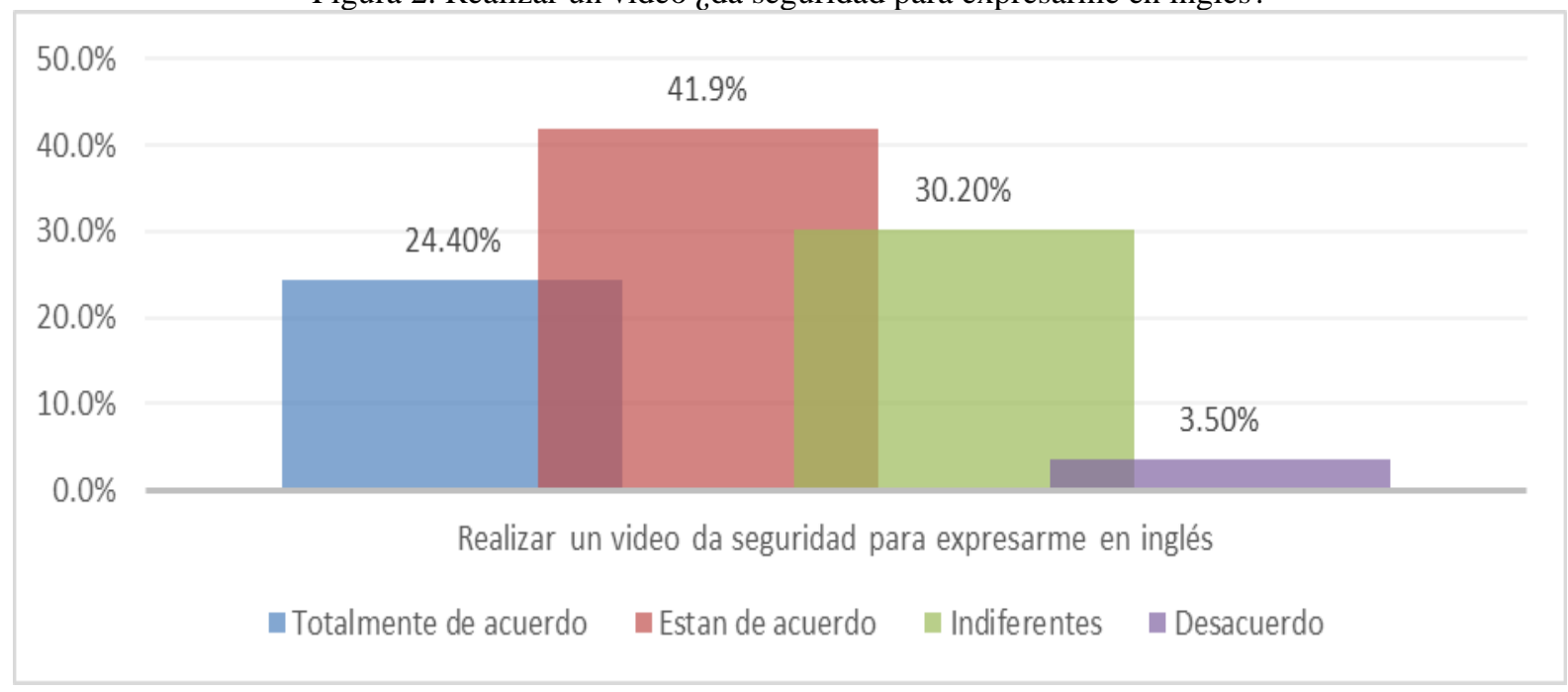

Cuando se les interrogó sobre si consideraban que al hacer un video memorizó el vocabulario aprendido, 36 sujetos (41.9\%) dijeron estar de acuerdo, 29 sujetos (33.7\%) dijeron estar totalmente de acuerdo y solo 3 sujetos (3.5\%) manifestaron su desacuerdo y 18 sujetos (20.9\%) son indiferentes.

Al preguntarles sobre si la realización del video era significativa para su aprendizaje del idioma el $44.2 \%$ (38 sujetos) mencionaron estar de acuerdo, el 27.9\% (24 sujetos) dijeron estar totalmente de acuerdo, solo el $3.5 \%$ (3 sujetos) mencionaron su desacuerdo y $24.4 \%$ (21 sujetos) son indiferentes. 
En la pregunta sobre si les gustaría tener más actividades para realizar otros videos, el 36\% (31 sujetos) dijeron estar de acuerdo, 34.9\% (30 sujetos) mencionaron ser indiferentes y solo el $12.8 \%$ mencionaron estar totalmente de acuerdo.

Para la pregunta de si los videos en idioma ingles les permiten descubrir nuevos aspectos en el aprendizaje, 35 sujetos (40.7\%) mencionaron estar de acuerdo, 25 sujetos (29.1\%) dijeron estar totalmente de acuerdo, solo 6 sujetos (7.0\%) mencionaron su desacuerdo.

Para la pregunta si consideraban que su participación oral en el aula aumento significativamente el 45.3\% (39 sujetos) dijeron estar de acuerdo, 30.2\% (26 sujetos) manifestaron su indiferencia, solo el $18.6 \%$ (16 sujetos) mencionaron estar totalmente de acuerdo.

Cuando se les preguntó sobre si la elaboración del video mejoro su conversación el 52.3\% (45 sujetos) dijeron estar de acuerdo, 30.2\% (26 sujetos) mencionaron ser indiferentes, $12.8 \%$ (11 sujetos) totalmente de acuerdo solo el 4.7\% (4 sujetos) mencionaron su desacuerdo.

Estos resultados favorecen la investigación, Waked Hernández (2016, p. 106) al realizar una investigación similar dentro de la cual analiza el uso del video como medio tecnológico para mejorar el aprendizaje en una lengua extranjera, evidencio que los aprendices de español como lengua extranjera demostraron lo aprendido al aplicar los medios tecnológicos, entre ellos el video, en diversas actividades escritas y orales.

El $80.2 \%$ de los alumnos consideraron la elaboración del video como una herramienta útil, así mismo para Azuara Guillén, Fernández Iglesias, López Torres, et al. (2017, p. 351) al realizar una investigación sobre: vídeos cortos realizados por los alumnos como recurso docente, destaca que el 96\% de los alumnos que participaron en el proyecto manifestaron una satisfacción con la actividad, mientras que el $79 \%$ de los alumnos concuerdan que se tiene una mejor asimilación de los conceptos que si realizaban otras actividades.

Si lugar a dudas el video como recurso de aprendizaje debe ser más utilizado por los docentes, ya que constituye un medio actual que manejan los jóvenes, sobre todo en sus redes sociales. Para ellos usar el celular para hacer videos les ayuda para practicar el vocabulario, la pronunciación y darles más seguridad.

\section{CONCLUSIONES}

De acuerdo con los resultados obtenidos en las encuestas del diagnóstico aplicado, podemos observar que la mayoría de los estudiantes, 73 sujetos están de acuerdo en que su profesor promueve la elaboración de videos en el aula y 69 de ellos consideran que elaborar el video si es una herramienta útil en el aprendizaje del idioma inglés. 
El objetivo de la investigación se logra ya que se pudo Identificar que la elaboración de videos es aceptado y aprobado como un recurso de aprendizaje en la enseñanza del idioma inglés en el nivel medio superior de la UAC por los alumnos ya que más del $80 \%$ de los encuestados afirman que el realizar un video en ingles les ayuda a practicar su pronunciación, vocabulario y gramática. También 57 estudiantes consideran que realizar un video sí les da seguridad para expresarse en inglés. 55 sujetos consideran que su participación oral en el aula aumento significativamente, y más del 70\% afirman que la elaboración del video mejoro su conversación en Ingles.

También un punto importante es que 72 estudiantes están de acuerdo que es necesaria la actualización continua del profesor en el uso de recursos de Internet, (videos, audios y blog).

Con estos resultados podemos concluir que para los docentes es muy importante estar a la vanguardia, si se utiliza la tecnología para impartir clases, pero no se usa como método de aprendizaje para los estudiantes. La elaboración del video en la clase de Ingles logra los objetivos deseados al mejorar el listening y el speaking, como se requiere al aprender un idioma, inclusive en nuestro propio idioma, este recurso es significativo para un mejor desempeño oral y de pronunciación.

La elaboración de videos para el aprendizaje puede representar una actividad diferente para el alumno, lo cual es positivo, ya que "Resulta conveniente de vez en cuando variar la dinámica de la interacción en el aula de lengua extranjera, garantizándose así el mantenimiento de los procesos atencionales y motivacionales, imprescindibles para el desarrollo del proceso de aprendizaje lingüístico.” (Martínez, 2003).

Se sugiere realizar un análisis sobre los mecanismos de evaluación del video como recurso de aprendizaje, estableciéndose rubricas o listas de cotejo que ayuden al alumno y al docente para establecer y entender los criterios de evaluación. También se recomienda la capacitación de los docentes en la elaboración de videos usando los celulares.

Definitivamente los docentes debemos motivar a la realización de videos como proyectos frecuentes ya que el estudiante además de mejorar el aprendizaje del idioma inglés usa la creatividad, dinamismo y hasta sentido del humor para crear producciones originales que los ayuda a estar a la vanguardia y a promover sus sentidos y estilos de aprendizaje. 


\section{REFERENCIAS}

Almeida Aguilar, M.A., Jerónimo Yedira, R., Acosta de la Cruz, J. T., \& Ramos Méndez E. (2017). Los objetos de aprendizaje como herramientas de enseñanza y aprendizaje. Revista De la Alta Tecnología Y Sociedad, 9(1), 1-7.

Azuara Guillén, G., Fernández Iglesias, D. López Torrez A.M., et al (2017). Vídeos cortos realizados por los alumnos como recurso docente. Diferentes enfoques. Actas de las XIII Jornadas de Ingeniería Telemática, Valencia, España. en: https://pdfs.semanticscholar.org/fbdb/5a3d48f8299ba082bb7e81b21dba3eccf611.pdf

Bartolomé, P. A. (1987). Análisis de la producción y aplicación de programas audiovisuales didácticos. Disponible en:

http://www.tdx.cat/bitstream/handle/10803/2360/01.ARBP_1de11.pdf.txt?sequence=22

Blanco Sánchez, M. I. (2012). Recursos didácticos para fortalecer la enseñanza-aprendizaje de la economía. Disponible en: https://uvadoc.uva.es/bitstream/10324/1391/1/TFM-E\%201.pdf

Corpas, J. (2004). La utilización del vídeo en el aula de E/LE el componente cultural. Difusión.

Del Moral, M. E., \& Cernea, D. A. (2005). Diseñando Objetos de Aprendizaje como facilitadores de la construcción del conocimiento. In II Simposio Pluridisciplinar sobre Diseño, Evaluación y Descripción de Contenidos Educativos Reutilizables. Recuperado de: http://www.uoc.edu/symposia/spdece05/pdf/ID16.pdf

Diccionario de la Lengua Española (DLE) (2017). Video. Disponible en: https://dle.rae.es/?id=bm7DOSs. Consultado en 14/mayo/2017.

Grisolía, M. (2015). Web del profesor. Disponible en:

http://webdelprofesor.ula.ve/humanidades/marygri/recursos.php

Martínez, J. D. (2003). Hacia una enseñanza de lenguas extranjeras basada en el desarrollo de la interacción comunicativa. Didáctica (Lengua y Literatura), 139-160.

Organización de la Naciones Unidas para la Educación, la Ciencia y la Cultura, UNESCO 2008. Estándares de competencias en Tic para docentes. Obtenido de: http://eduteka.icesi.edu.co/pdfdir/UNESCOEstandaresDocentes.pdf

Waked Hernández, M. (2016). El uso de los medios tecnológicos para mejorar el aprendizaje del español como lengua extranjera. Horizontes Pedagógicos, 18(1), 96-107. Recuperado a partir de https://horizontespedagogicos.ibero.edu.co/article/view/18109 